University of Nebraska - Lincoln

DigitalCommons@University of Nebraska - Lincoln

Faculty Publications: Materials Research

Science and Engineering Center

Materials Research Science and Engineering

Center

2007

\title{
Nonvolatile Two-Terminal Molecular Memory
}

\author{
Jason Snodgrass \\ University of Nebraska Omaha, Omaha, NE \\ Glen Kennedy \\ University of Nebraska Omaha, Omaha, NE \\ Wai-Ning Mei \\ University of Nebraska at Omaha, physmei@unomaha.edu \\ Renat F. Sabirianov \\ University of Nebraska at Omaha, rsabirianov@mail.unomaha.edu
}

Follow this and additional works at: https://digitalcommons.unl.edu/mrsecfacpubs

Part of the Materials Science and Engineering Commons

Snodgrass, Jason; Kennedy, Glen; Mei, Wai-Ning; and Sabirianov, Renat F., "Nonvolatile Two-Terminal Molecular Memory" (2007). Faculty Publications: Materials Research Science and Engineering Center. 87. https://digitalcommons.unl.edu/mrsecfacpubs/87

This Article is brought to you for free and open access by the Materials Research Science and Engineering Center at DigitalCommons@University of Nebraska - Lincoln. It has been accepted for inclusion in Faculty Publications: Materials Research Science and Engineering Center by an authorized administrator of DigitalCommons@University of Nebraska - Lincoln. 


\title{
Nonvolatile Two-Terminal Molecular Memory
}

Jason Snodgrass ${ }^{1}$, Glen Kennedy ${ }^{1}$, Wai-Ning Mei ${ }^{1}$, and Renat Sabirianov ${ }^{1,2}$

${ }^{1}$ University of Nebraska Omaha, Omaha, NE, 68182-0226

${ }^{2}$ Nebraska Center for Materials and Nanotechnology, Lincoln, NE, 68588-0111

\begin{abstract}
We propose a nonvolatile two-terminal memory device with two resistance states based on the molecular tunnel junctions. This tunnel junction is composed of one or a few monolayers of polar molecules sandwiched between two electrodes made of materials with different screening length. As a prototype model system we study a rare earth endohedral metallofullerene molecule with reversible dipole moment sandwiched between metal and semiconducting electrodes, forming a double barrier junction. We use the Thomas-Fermi model to calculate the potential profile across the device. Calculated tunneling conductance through the proposed structure changes by order of magnitude upon the reversal of the dipole orientation (due to the applied voltage). This effect originates from the difference in potential profiles seen by tunneling electrons for two opposite dipole orientations.
\end{abstract}

\section{INTRODUCTION}

Resistive switching in metal-insulator heterostructures has recently attracted considerable attention because of their potential applications as two-terminal non-volatile memory where the resistance of a device can be switched and read by applying an external voltage [1,2]. Recently, similar switching has been observed in asymmetric metal/ferroelectric films [3] and theoretically predicted for ferroelectric tunnel junctions [4]. Advances in molecular electronics show possible resistive switching through conformational changes of the molecule, as well as by interface modifications.[5]

In this work we propose a new method for the resistive switching in a variety of systems where abrupt modification of the surface properties is possible. This method is based on selecting interfaces with the possibility of changing their charge states. This can occur due to the conformational changes to the molecule or the charge trapping on the interface, and, as a result, a modification of the conducting properties. In this work, we show that the abrupt change of the state at the interface due to the applied voltage leads to a sizable change in the tunneling current. This provides a two-terminal electrical control of the resistance, including the possibility of controllable switching from a high resistance state (HRS) to a low resistance state (LRS).

\section{THEORY}

The particular system we will consider is a tunnel junction representing a molecule sandwiched between two asymmetric electrodes. The possibility of using molecules as tunnel barriers has been actively studied in recent years both experimentally and theoretically. These results suggest that the ratio of the resistance states (HRS) and (LRS) upon switching is quite 
large. Recent experiments indicate that the electrical resistance switching in metal/molecule/metal junctions may not depend on the particular molecular specie but on the state of the interface and its modification with an applied electric field.[6] In our previous work we have predicted the existence of a giant electroresistance (GER) effect in ferroelectric tunnel junctions.[4]

Using a simple model we investigate how the change in potential profile caused by modification of the electric dipole of the molecule and the charge state of the interface produces a very large change in resistance. The resistance switching originates from the change in potential profile induced by the charges at the interface due to the different screening lengths in the electrodes with unequal charge densities.

In order to describe this effect quantitatively we consider a semi-infinite electrode placed in the half-space $z<0$ (layer 1), a molecular barrier of thickness $a$ (layer Mol), and another electrode of different material placed in the half-space $z>d$ (layer 2). In order to simulate the effect of the electrodes we will vary the carrier density in each electrode separately. The left electrode will represent a semiconductor with the lower concentration of carriers and the right electrode will be a good metal, forming a S/Mol/M structure. The details of the calculation will depend on the choice of the particular molecule and selected method of electrostatic potential calculations, but the qualitative picture of the electrostatic in the proposed tunnel junction will not change and it is applicable to a wide variety of polar molecules.

We use the Thomas-Fermi model to describe the electronic structure of the system, its potential, and its charge distribution. Since the Fermi energy, $E_{F}$, is constant throughout the structure, the electronic potential in the right electrode, $V_{2}$, with respect to $V_{1}$ is controlled by the carrier concentrations in the left electrode, $n_{1}$, and in the right electrode, $n_{2}$. The molecular barrier is represented by a double rectangular potential of height $U$ with respect to $E_{F}$, which implies that the work functions of the two electrodes are assumed to be the same. The lateral dimension of the molecule is $1 \mathrm{~nm}$ (this represents the outer diameter of $\mathrm{C}_{60}$ or $\mathrm{C}_{82}$ ). The thickness of these barriers, $d$, was varied from $0.5 \mathrm{~nm}$ to $1 \mathrm{~nm}$. This potential mimics molecular junctions where the interfaces are highly resistive while the molecule is "conductive". As a particular example of a possible molecular tunnel junction (MTJ) we will use gadolinium endohedral metallofullerene. First-principle calculations of the electronic structure of molecular junctions (such as endohedral metallofullerene Gd@C60) show that the charge distribution is not uniform throughout the molecule. The endohedral atom transfers most of its valence electrons to the carbon cage atoms it is closest to. Natural population analysis shows that $2.43 \mathrm{e}$ out of 3 leave the $\mathrm{Gd}$ atom in Gd@C60 rendering a dipole moment of about 2Db. [7]. As a result, the electrostatic potential is not uniform throughout the molecule. Its variation on average, however, is very small. A major drop in the electrostatic potential occurs at the interfaces. The dipole position is off-center in the ehdohedral fullerenes. This dipole position changes upon reversal of the dipole direction. The charge distribution in such a system along the axis of symmetry is represented in Figure 1(a). The dipole is represented by two localized areas of positive and negative charge, $\sigma$. The dipole center is shifted towards left electrode. The dipole produces an electric field which is screened by surface charge densities, $\sigma_{S}$ and $\sigma_{S}^{\prime}$, in the asymmetric electrodes. A simple dipole approximation would give us variation of the electrostatic potential across the tunnel junction along the axis of symmetry as represented in Figure 1 (b). Assuming that the molecule can be represented as a dipole at a distance $d$ from the metal surface one can solve the linearized Thomas-Fermi model, described by $\nabla^{2} \varphi=\delta^{-2} \varphi$, exactly.[8] The potential in the metal, with cylindrical symmetry and boundary at $\mathrm{z}=0$ is 


$$
\varphi=2 p \delta \int k J_{0}(k \rho) e^{\sqrt{k^{2}+\delta^{-2}} z-k d} g(k) d k
$$

Here $\delta$ is the Thomas-Fermi screening length in the metal electrode, $p$ is a dipole moment, $J_{0}$ is a Bessel function, $k$ is a wave vector, and $\rho$ is a polar coordinate ( $\rho=0$ in Figures 1 and 2 ). The function $g(k)$ is determined from the boundary conditions. The short circuit condition is applied here as both left and right electrode potentials $\varphi(z) \rightarrow 0$ when $z \rightarrow \infty$. The screening length at zero applied potential is

$$
\delta^{-2}=4\left(\frac{3 n}{\pi}\right)^{1 / 3} \frac{m e^{2}}{\hbar^{2}},
$$

where $m$ is the effective of the right/left electrode, and $n$ is the charge density in the electrode.
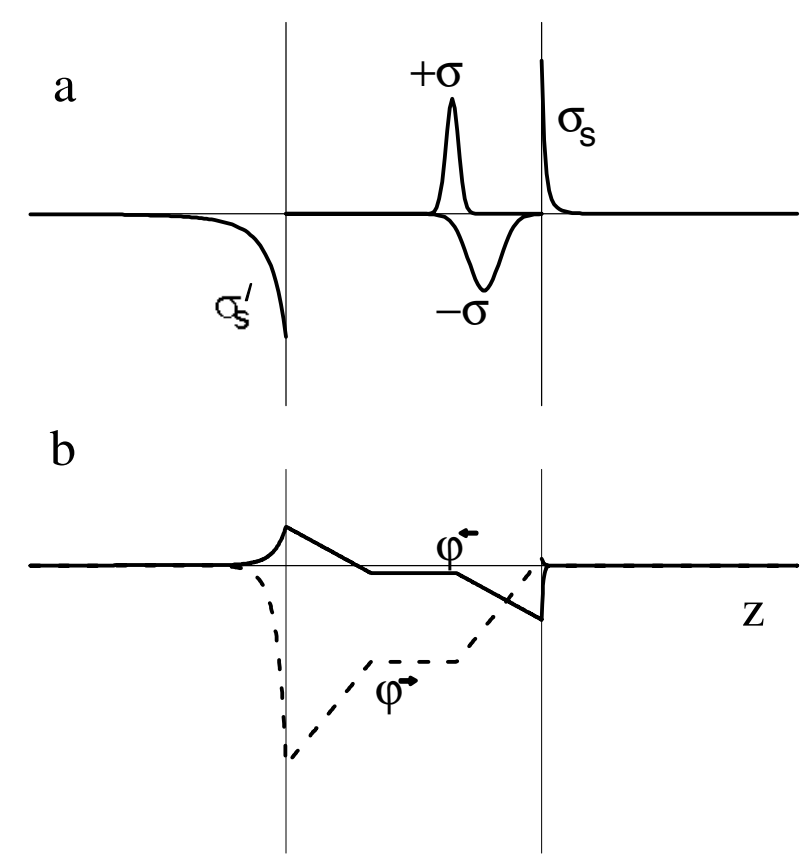

Fig.1 Electrostatics of a S/Mol/M junction: (a) charge distribution and (b) the respective electrostatic potential profile (solid line). It is assumed that semiconductor in layer 1 and metal in layer 2 electrodes have different screening lengths $\left(\delta_{1}>\delta_{2}\right)$ which lead to the asymmetry in the potential profile. The dashed line in (b) shows the potential when both the dipole direction and the dipole position are switched, resulting in the reversal of the screening charge sign.

Fig. 2 shows the overall potential profile, $V(z)$, which is the sum of the electrostatic potential, $\varphi(z)$, the electronic potential in the electrodes, which controls the position of the bottom of the bands, and the rectangular potential profile discussed above for the molecular tunnel junction. An important observation which follows from Figure 1 is that when the dipole is shifted closer to the left electrode in the molecular barrier, the effective width of the tunneling barrier can be different compared to the case when the $\mathrm{Gd}$ atom is shifted towards the right electrode. This occurs if the magnitude of the electrostatic potential at the interface, $\varphi_{1} \equiv|\varphi(0)|$, is larger than the 
Fermi energy with respect to the bottom of the band, that is $E_{F}-V_{1}-\phi_{1}>0$. Therefore, the conductance will be different in these two states: a thinner barrier case will correspond to a lower resistance state and a thicker barrier will correspond to a higher resistance state.

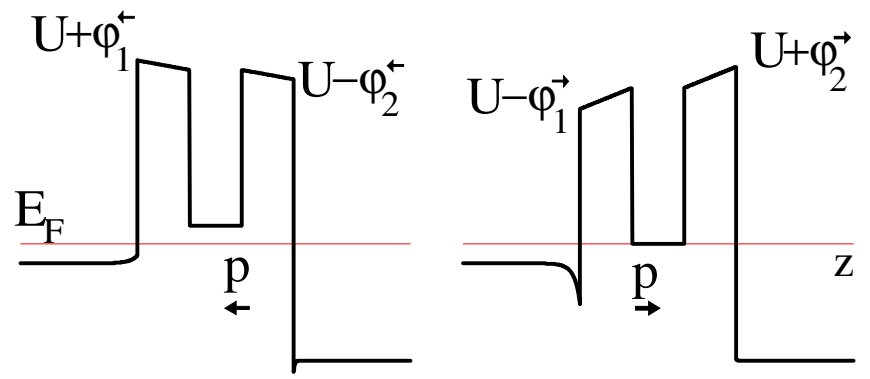

Fig. 2 Potential profile $V(z)$ in a S/Mol/M tunnel junction for the electric dipole pointing to the left (a) and to the right (b). The horizontal solid line denotes the Fermi energy, $E_{F}$.

In order to make these arguments quantitative, we calculate the conductance of the molecular tunnel junction using the Landauer formula,

$$
G=\frac{2 e^{2}}{h} \int \frac{d k_{\|}}{(2 \pi)^{2}} T\left(E_{F}, k_{\|}\right) .
$$

Here $G$ is the conductance per unit area, $T\left(E_{F}, k_{\|}\right)$is the transmission coefficient evaluated at the Fermi energy, $E_{F}$, for a given value of the transverse wave vector $k_{\|}$. The transmission coefficient is obtained by solving numerically the Schrödinger equation for an electron moving in the potential $V(z)$ by imposing a boundary condition of the incoming plane wave normalized to unit flux density and by calculating the amplitude of the transmitted plane wave. The effective masses of carriers in the electrodes are set to be a free electron mass, and the dielectric permittivities are the permittivity of vacuum for simplicity. The Fermi energy in metallic layer 2 is fixed at $E_{F}=3.5 \mathrm{eV}$ (with respect to the bottom of the band), resulting in a screening of $\delta_{2}=0.07 \mathrm{~nm}$, typical for good metals.

\section{DISCUSSION}

Fig. 3a shows the variation of the magnitude of the calculated electrostatic potential at the surface along the axis of symmetry as function of screening length for three different distances between the dipole and the surface of the metal electrode. The magnitude of the potential is increasing with the increase of the screening length (and eventually saturating), and with the reduction of the distance between the dipole and the metal surface.

The asymmetry of the screening potential at the left and right electrodes controls the ratio of conductance upon the reversal of the dipole moment of the molecule. There are several mechanisms responsible for the conductance change upon reversal of dipole. (i) The average potential barrier heights seen by the transport electron when tunneling with the dipole switched to the left are $\mathrm{U}_{\mathrm{L}, 1}=\mathrm{U}+\left(3 \varphi_{1}-\varphi_{2}\right) / 4$ and $\mathrm{U}_{\mathrm{L}, 2}=\mathrm{U}+\left(\varphi_{1}-3 \varphi_{2}\right) / 4$, for the first and second barriers, respectively. While, tunneling through the barrier with the dipole to the right it sees potential 
barriers $\mathrm{U}_{\mathrm{R}, 1}=\mathrm{U}+\left(\varphi_{2}-3 \varphi_{1}\right) / 4$ and $\mathrm{U}_{\mathrm{R}, 2}=\mathrm{U}+\left(3 \varphi_{2}-\varphi_{1}\right) / 4$. Besides direct consequences for tunneling, the difference in barrier heights in the double barrier junction may cause charging of the molecule depending on the orientation of the dipole. (ii) The existence of the electric field in the system shifts molecular energy levels relative to the states of the electrodes. Such shifts may alter tunneling considerably, especially if it is close to the resonant tunneling when there is a molecular state with energy close to the Fermi energy. (iii) When the Fermi energy is small enough that the screening potential in the electrode effectively thickens the barrier. Since the tunneling coefficient decays exponentially with the thickness of the barrier, it may cause a large electroresistance ratio. The latter effect may be realized in narrow band semiconductor electrodes where the screening potential exceeds the Fermi energy in the semiconductor.

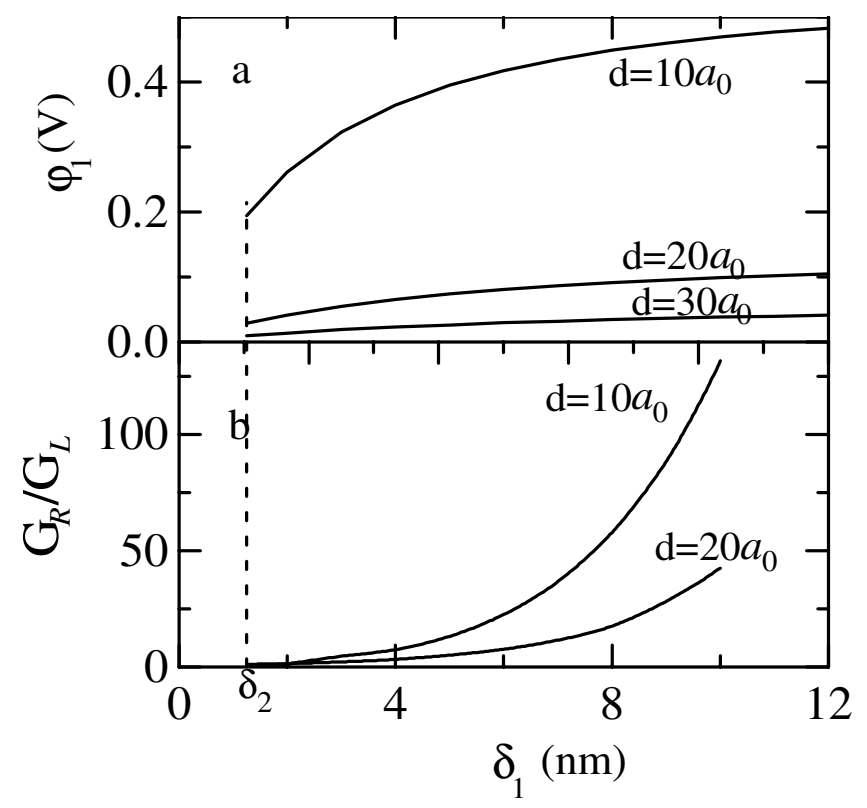

Fig. 3 (a) Electrostatic potential height on the surface along the axis of symmetry due to a dipole moment at the distance $d$ from the surface. (b) $G_{R} / G_{L}$ - ratio of conductances through a S/Mol/M tunnel junction upon dipole switching as a function of screening length. $\mathrm{d}$ represents the spacing between the molecule and electrodes. The vertical dashed line corresponds to the symmetric electrode case with $\delta_{1}=\delta_{2}$, and therefore, no conductance difference is predicted. $a_{0}$ is a Bohr radius.

Figure $3 \mathrm{~b}$ shows the calculated ratio of conductance with the dipole switched to the right, $G_{R}$, and to the left, $G_{L}$, as function of the screening length for two geometries of the molecular junction. We have used $\mathrm{U}=0.5 \mathrm{eV}$ in our calculations which is the representative barrier for a variety of insulating media. The conductance ratio decreases with the increase of $U$ but remains large for $\mathrm{U}$ up to $5 \mathrm{eV}$ which corresponds to a work function of metals such as Pt.

It can be observed that the geometry with shorter spacing between the molecule and electrode has a larger electroresistance ratio. This can be explained by a larger screening potential at the interface as can be seen from fig. 3a. It may exceed a factor of 100 when the screening length is larger than $1 \mathrm{~nm}$. This may explain the large resistance switching in the molecular junction observed experimentally by Yasutake et al. for the STM probe/ vacuum/ single Tb@C82 molecule/ alkanethiol SAM/ $\mathrm{Au}(111)$ structure. [5]. 


\section{CONCLUSIONS}

In conclusion, we have proposed a device which permits resistance switching of a molecular tunneling junction by reversing the dipole direction and dipole position of a molecule. We have modeled a particular case of metal atom in endohedral metallofullerene such as $\mathrm{Gd}_{0} @ \mathrm{C}_{60}$ where dipole reversal is achieved by the applied voltage. This effect can be used for designing twoterminal non-volatile memory where the switching and reading are performed with the same terminals. We hope that our results will stimulate experimental studies of the molecular tunnel junctions with conformational changes.

\section{ACKNOWLEDGMENTS}

This research was supported by Nebraska Research Initiative (No. 4132050400, No. 2132151032), NSF “QSPINS" MRSEC (DMR 0213808). The authors thank Dr. M. Zhuravlev,

E.Y. Tsymbal, and G.Li for fruitful discussions. This work was completed utilizing the Research Computing Facility of the University of Nebraska - Lincoln.

\section{REFERENCES}

1. A. Baikalov, Y. Q. Wang, B. Shen, B. Lorenz, S. Tsui, Y. Y. Sun, Y. Y. Xue, and C. W. Chu, Appl. Phys. Lett. 83, 957 (2003).

2. P. Weiss, Science News 167, 363 (2005).

3. J. Rodríguez Contreras, H. Kohlstedt, U. Poppe, R. Waser, C. Buchal, and N. A. Pertsev, Appl. Phys. Lett. 83, 4595 (2003).

4. M. Ye. Zhuravlev, R. F. Sabirianov, S. S. Jaswal, and E. Y. Tsymbal, Phys. Rev. Lett. 94, $246802(2005)$.

5. Y. Yasutake, Z. Shi, T. Okazaki, H. Shinohara, and Y. Majima, NanoLetters 5, 1057 (2005).

6. D. R. Stewart, D. A. A. Ohlberg, P. A. Beck, Y. Chen, and R. Stanley Williams, J. O. Jeppesen, K. A. Nielsen, and J. Fraser Stoddart, NanoLetters 4, 133 (2004).

7. J. Lu, W.N. Mei, Y. Gao, X. Zeng, M. Jing,G. Li, R. Sabirianov, Z. Gao, L. You, J. Xu, D. Yu, H. Ye, Chemical Physics Letters 425, 82-84 (2006).

8. N.W. Ashcroft and N. D. Mermin, Solid State Physics(Saunders College Publishing, New York, 1976), p. 340. 